DOI: 10.25100/eg.v0i21.11291

Espacios y Territorios

\title{
Susceptibilidad a procesos de remoción en masa: Aproximación metodológica para la construcción de un índice de amenaza en Álvaro Obregón-Ciudad de México
}

\section{Susceptibility to landslides: Methodological approach for the construction of a hazard index in Álvaro Obregón-Mexico City}

\author{
María Alejandra Cortés Ortiz \\ Doctora (C) Universidad Nacional Autónoma de México \\ malejacortesortiz@gmail.com | 0000-0002-7030-3061 \\ José Ramón Hernández Santana \\ Doctor en Ciencias Geográficas. Universidad Nacional Autónoma de México. \\ hernandezsantanajr@hotmail.com, jhsantana@posgrado.unam.mx | 0000-0002-1375-2776 \\ Adrián Guillermo Aguilar Martínez \\ Doctor en geografía. Universidad Nacional Autónoma de México. \\ $\underline{\text { adrianguillermo1@gmail.com | 0000-0003-0376-2492 }}$
}

Para citar este artículo: Cortés Ortiz, M. A., Hernández Santana, J. R. y Aguilar Martínez, A.G. (2021). Susceptibilidad a procesos de remoción en masa: Aproximación metodológica para la construcción de un índice de amenaza en Álvaro Obregón-Ciudad de México. Entorno Geográfico, (21), 47-76.

https://doi.org/10.25100/eg.v0i21.11291

\section{Resumen}

Este artículo presenta una aproximación metodológica para la evaluación de la amenaza, por medio de un índice que determina la susceptibilidad por procesos de remoción en masa (PRM) y sus niveles de amenaza en los periodos de lluvia, a partir de un método mixto (cualitativo y cuantitativo), derivado de una investigación doctoral, sobre la configuración del riesgo en espacios periurbanos de la Ciudad de México. Se empleó una sumatoria multifactorial, que combina parámetros geomorfológicos y morfométricos determinantes y detonantes en la ocurrencia de PRM, aplicado en la Alcaldía de Álvaro Obregón- al poniente de la Ciudad de México, dada su alta ocurrencia de desastres en el país. El resultado de factores como: la litología, el uso del suelo y vegetación, la categoría geomorfológica, pendiente, geometría de laderas, energía del relieve y como detonante, la lluvia, por medio de la precipitación máxima histórica mensual de la mitad lluviosa del año (mayo-octubre), determinaron que, más del 50\% del territorio es susceptible a PRM, particularmente en los tres meses con mayor intensidad de lluvias. Este resultado busca servir 
de base para la discusión teórica y metodológica en el conocimiento de la evaluación multicriterio de la amenaza, con un aporte en la construcción de escenarios de riesgo y su gestión.

Palabras clave: Amenaza, Susceptibilidad, Procesos de remoción en masa, Índice, Suma de rásteres (SIG) y Álvaro Obregón-Ciudad de México.

\begin{abstract}
This article presents a methodological approach for the evaluation of the hazard, by means of an index that determines the landslides for susceptibility and its hazard levels in the rainy periods, based on mixed method (qualitative and quantitative), derived from a Doctoral research, on the configuration of risk in peri-urban spaces in Mexico City. A multifactorial summation was used that combines determining and triggering geomorphological and morphometric parameters in the occurrence of MRP, applied in the borough of Alvaro Obregon- to the west of Mexico City, given its high occurrence of disasters in Mexico. Diverse factors were evaluated such as: lithology, land use and vegetation, geomorphological categories, slope, hillside geometry, relief energy, and as a trigger, rain, by means of the maximum historical monthly rainfall of the rainy half of the year (may-october). The result determined that more than $50 \%$ of the territory is susceptible to MRP, particularly in the three months with the highest intensity of rainfall. This result serves for the theoretical and methodological discussion for the knowledge of the multi-criteria evaluation of hazards as a main contribution in the construction of risk scenarios and their management.
\end{abstract}

Keywords: Hazard, Susceptibility, Landslide, Index, Raster Sum (GIS) \& Alvaro ObregonMexico City.

Recibido: 30 de junio de 2020

Aceptado: 05 de noviembre del 2020

\title{
Introducción
}

Los procesos de remoción en masa (PRM), conocidos también como procesos gravitacionales, movimientos de terreno o movimientos de ladera, o coloquialmente: deslaves o derrumbes (Alcántara, 2010), son fenómenos implicados en la configuración del relieve terrestre, principalmente en la evolución de los paisajes de montaña (Korup et al., 2010). Estos se clasifican en: en caídas o desprendimientos, vuelcos o desplomes, deslizamientos, expansiones laterales, flujos y movimientos complejos, que involucran el movimiento de material formador de laderas o 
masas de suelo (detritos y roca); condicionados por factores internos y externos, propiciando una amplia gama de diversidad en el paisaje como producto de la interacción endógena y exógena de la dinámica terrestre, así como por las actividades antropogénicas (Alcántara, 2000; Alcántara y Murillo, 2008).

Estos procesos son determinados por: a) cambios en la geometría del terreno, ya sea de forma natural o por actividad humana y b) los relacionados con la composición físico-química de los materiales que las constituyen, su disposición y resistencia a presiones del exterior. Los factores detonantes tienen relación con lluvias torrenciales, sismos o erupciones volcánicas (Alcántara, 2000), siendo un significativo riesgo ante la exposición de la población susceptible a sufrir pérdidas humanas y daños, dada cualquiera de sus manifestaciones (Froude y Petley, 2018).

Para la identificación y evaluación de la amenaza por PRM, se requiere localizar las áreas inestables por tipo de movimientos, estableciendo los factores determinantes, relacionados con la estabilidad y su contribución a la inestabilidad (Pardeshi, Autade y Pardeshi, 2013; Becerra y De Rurange, 2018), derivados de las condiciones intrínsecas o locales del terreno, es decir, establecer su susceptibilidad. Esto, en conjunción con los factores detonantes, determinará el nivel de peligro o amenaza. En ese sentido, se entenderá por amenaza por PRM, el grado de predisposición que tiene un lugar dado para que se genere un evento, en función de factores "determinantes o condicionantes” y “detonantes” (Ayala-Carcedo, 2002; Becerra y De Rurange, 2018).

Los factores determinantes, son aquellos que provocan que la ladera sea susceptible a deslizarse por sus características geológicas, inclinación de superficies (laderas e incluso bordes pronunciados de llanuras), morfología y morfometría del relieve, y actividades antrópicas; mientras que los factores detonantes, son los que provocan que la ladera se colapse, a causa de algún agente externo como precipitación (intensidad y cantidad), actividad sísmica, erupciones volcánicas, incremento de peso en la laderas, entre otros (Alcántara, 2000; Ayala, 2002).

Según lo documentado en revisiones de literatura sobre susceptibilidad y evaluación de la amenaza por PRM (Aristizábal, Martinez y Vélez, 2010; Pourghasemi, Teimoori, Panagos y Pradhan, 2018; Quesada-Román, Moncada-López, Paz-Tenorio, 2018; Reichenbach, Rossi, Malamud, Mihir, Guzzetti, 2018) y literatura más reciente (Shano, Raghuvanshi y Meten, 2020; Xiao, Segoni, Chen, 
Yin y Casagli, 2020), diversos autores han propuesto una amplia variedad de metodologías (modelos e índices) para estimar amenaza por los PRM, basadas en la toma de decisiones por expertos (heurística), otras que combinan métodos cualitativos y cuantitativo (mixto o semicuantitativo), métodos estadísticos y análisis multicriterio, aplicadas en diferentes escalas espaciales (local, nacional, regional y global), usando y combinando diferentes técnicas como: la detección remota, fotografías áreas y sistemas de información geográfica (SIG); e integrando diferentes variables explicativas entorno a los factores determinantes y detonantes; aunque no hay una estandarización al respecto, todos estos trabajos buscan contribuir en la identificación y evaluación de la amenaza para la prevención de desastres y mitigación del riesgo asociado a estos eventos.

En México, estas metodologías se han aplicado en diversas escalas, particularmente en los estados de Chiapas (Hernández Moreno, 2011 y Paz Tenorio, González, Gómez y Velasco, 2017); Puebla, en los municipios de Zapotitlán (Borja Baeza y Alcántara, 2004), Zacapoaxtla (Hernández Mena, 2008) y Pahuatlán (Murillo García y Alcántara, 2015), y en Jalisco, en el municipio de Puerto Vallarta-Jalisco (Muñiz Jauregui y Hernández, 2012). De estos trabajos se hará mención específica de tres de estas contribuciones.

Paz Tenorio y colaboradores (2017), realizaron un ajuste metodológico a un mapa de amenazas por procesos de remoción en masa (PRM), realizado en el 2012, en una ladera al sur de Tuxtla Gutiérrez en Chiapas, a partir del método heurístico en combinación de análisis multicriterio, considerando cinco factores (geología, desniveles, densidad de drenaje, edafología y uso del suelo y vegetación), con lo que determinaron cinco niveles de amenaza en esta zona urbana, calculando alrededor del $12 \%$ de la población expuesta a estos eventos.

Murillo García y Alcántara Ayala (2015), realizaron un análisis y mapeo de susceptibilidad a PRM en el municipio de Pahuatlán en Puebla, utilizando técnicas estadísticas multivariadas (regresión logística, discriminante lineal, red neuronal y análisis discriminante cuadrático); los resultados se validaron con un inventario de PRM ocurridos entre 1994 y 2010, la calidad del modelo se evaluó por medio de métodos estadísticos (curvas ROC y de Cohen y Coeficiente Kappa), y se determinó, que el ángulo de la pendiente y la unidad litológica fueron las variables con el mayor peso asociado con la ocurrencia de PRM en la zona de estudio. 
Por su lado, Muñíz-Jauregui y Hernández Madrigal (2012), realizaron una zonificación de procesos de remoción en masa en la ciudad de Puerto Vallarta, Jalisco, combinando el análisis multicriterio y el método heurístico. En su elaboración, consideraron diez factores condicionantes o capas temáticas asociados a la inestabilidad (mapa-inventario), litología, pendiente, radiación solar, cortes en talud, zonas inestables por caminos, asentamientos humanos, distancia a morfolineamientos, áreas de desmonte y concentración de escurrimientos superficiales. Obtuvieron una zonificación con seis grados de susceptibilidad y se determinó que el $69 \%$ del municipio presenta niveles de media a muy alta susceptibilidad.

La importancia en la identificación y evaluación de la amenaza, por medio de la susceptibilidad a PRM (Pardeshi et al., 2013), toma valor cuando se suma el incremento poco regulado de los asentamientos humanos en laderas o zonas montañosas propensas a sufrir este tipo de procesos (Nojavan M., Salehi E. y Omidvar B., 2017), particularmente por eventos geológicos e hidrometeorológicos extremos como las lluvias de tormentas severas, lo cual está condicionado por la nula planeación de la expansión y crecimiento en asentamientos humanos y por las características socioeconómicas de la población, sobre todo en países de bajos y medianos ingresos (Cui, Cheng, Choi, Jin y Kargel, 2019; Holcombe, Beesley, Vardanega, 2016), combinación que magnifica el riesgo por PRM.

El incremento de desastres en las últimas décadas ${ }^{1}$, causados por las propias dinámicas planetarias, regionales y hasta locales, las notables tendencias desfavorables en la variabilidad y cambio climáticos, a escala global, y las formas de ocupación de la población en las zonas expuestas a eventos naturales con elevados grados vulnerabilidad y riesgo, afectará a las poblaciones más vulnerables, dada la degradación del suelo, según expone el Grupo Intergubernamental de Expertos sobre el Cambio Climático en su último reporte "Climate Change and Land", en un informe especial sobre cambio climático, desertificación, degradación de la tierra, gestión sostenible de la tierra, seguridad alimentaria, y flujos de gases de efecto invernadero en ecosistemas terrestres (IPCC, 2019).

\footnotetext{
${ }^{1}$ Desastres reportados en la Base de datos EM-DAT del Centre de Recherche sur l'Epidémiologie des Desastres (CRED). Brussels: Université Catholique de Louvain; 2016. http://www.emdat.be Consultada en junio de 2020.
} 
Este incremento de desastres, se manifiesta en las interrupciones funcionales de las poblaciones o de su desarrollo, por medio de pequeños, medianos y grandes desastres (Mansilla, 2001), así como, por la carga económica para las administraciones locales, condición agravada por la vulnerabilidad de países, regiones y/o localidades con un nivel económico limitado, el cual dificulta la rápida recuperación e incluso la preparación adecuada para la gestión del propio desastre (Borja Baeza y Alcántara, 2004, p. 8). Dentro del contexto nacional, en México, los desastres hidrometeorológicos, seguidos por los geofísicos, son los que más pérdidas económicas han ocasionado en el periodo de 1990 a 2016, por un monto de 35,322 millones de dólares (Abeldaño Zúñiga y González Villoria, 2018, p. 3).

En este sentido, la estimación de la susceptibilidad a PRM, como base para la identificación y evaluación de la amenaza o el peligro, son pieza fundamental para establecer relaciones entre los distintos componentes que influyen en los niveles de riesgo y, por ende, en los desastres ocasionados por estas amenazas, siendo útil en la generación de cartografía temática (Hansen, 1984; Parise, 2000; van Westen et al., 2006, citados por Alcántara Ayala y Murillo García, 2008, p. 48). Aunque los mapas de susceptibilidad no son el mejor enfoque para enfrentar todos los tipos de amenazas naturales, en términos de PRM, son ampliamente usados y efectivos (Pourghasemi, et al., 2018). Por tanto, en este trabajo se presenta una aplicación metodológica de la susceptibilidad a procesos de remoción en masa, detonados por precipitación intensa ${ }^{2}$, como un aporte en la comprensión y gestión del riesgo, por medio de un índice que se representa espacialmente en un mapa/zonificación en cinco niveles de susceptibilidad.

La aplicación y validación de esta metodología, se llevó a cabo con datos e información de la Alcaldía Álvaro Obregón (AAO), localizada al sur poniente de la Ciudad de México (CDMX), en las coordenadas $19^{\circ} 21^{\prime} 23^{\prime \prime} \mathrm{N} 99^{\circ} 14^{\prime} 10^{\prime \prime} \mathrm{O}$ (Figura 1). Esta es la tercera demarcación más grande en población, con 749,982 habitantes (INEGI, Conteo Poblacional, 2015), distribuidos en 8,114.01 ha $\left(81 \mathrm{Km}^{2}\right)$. Autoridades en materia de Gestión del Riesgo, consideran a este municipio como la zona

\footnotetext{
${ }^{2}$ Esta propuesta, deriva de la investigación Doctoral en Geografía de la Universidad Nacional Autónoma de México, sobre "la configuración del riesgo en espacios periurbanos de la Ciudad de México", dirigida y asesorada por los Doctores: Adrián G. Aguilar-Martínez, Elizabeth Mansilla y José Ramón Hernández Santana de la Universidad Nacional Autónoma de México (UNAM).
} 
de mayor riesgo asociado a eventos naturales y desastres, no sólo de la CDMX, sino de toda la República Mexicana³.

Lo anterior, porque AAO se caracteriza por ser un territorio de fuertes contrastes físicos y sociales, en el que interactúan su heterogénea litología y relieve, modificado por las actividades antrópicas, particularmente por la extracción minera y la ocupación de barrancas y laderas, dado el explosivo crecimiento que tuvo la CDMX en las décadas del 60 y 70 (Lugo Hubp, Cordero Estrada y Zamorano, 1995; Linares Canto, 2012), configurando así, una zona de riesgo por la ocurrencia de procesos de remoción en masa, detonados por las lluvias intensas extremas y la actividad antrópica.

Aunado a ello, las condiciones socioeconómicas y culturales de AAO también son heterogéneas. Alrededor de 800 mil personas se distribuyen en 210 colonias (barrios), nueve pueblos, cuatro barrios y 73 asentamientos humanos irregulares (SEDUVI y Delegación Álvaro Obregón, 2011). Una minoría con alto poder adquisitivo, asentada en la zona baja o plana de la Alcaldía o en consolidados condominios horizontales o verticales en algunas zonas de barranca; mientras la mayoría de bajos recursos económicos cohabitan en viviendas que presentan múltiples deficiencias en su estructura, instalaciones y servicios, que en su mayoría fueron autoconstruidos con el sistema tradicional de tequios o faenas comunitarias (González Rivera, 2018; Alcaldía Álvaro Obregón y Protección Civil, sin fecha).

Predomina la ocupación en el suelo de conservación ${ }^{4}$, en áreas de valor ambiental y polígonos federales como barrancas, líneas de alta tensión y vías de ferrocarril, invadidos por agrupaciones sociales y por desarrolladores inmobiliarios, que no se han apegado al uso de suelo establecido dentro del Programa de Desarrollo Delegacional Urbano (PDDU), documentado en el Programa de Trabajo 2018-2021 de la Dirección de Protección Civil y Zonas de Alto Riesgo de AAO (Alcaldía Álvaro Obregón y Protección Civil, sin fecha).

\footnotetext{
${ }^{3}$ Según revela el Atlas de Peligros y Riesgos de la Ciudad de México (Secretaria de Protección Civil, 2014), Actualización de los Mapas de Riesgo de Álvaro Obregón. http://atlas.cdmx.gob.mx/mapas/MR_Alvaro_Obregon.pdf;http://www.atlas.cdmx.gob.mx/atlas2014.html;https://ww w.jornada.com.mx/2007/12/23/index.php?section=capital\&article=032n 1 cap

${ }^{4}$ CDMX, se divide en dos zonas administrativas: el suelo de conservación (SC) y el suelo urbano (SU). El primero se refiere a las zonas que, por sus características ecológicas, proveen servicios ambientales, necesarios para el mantenimiento de la calidad de vida de los habitantes de la ciudad, conforme a la Ley Ambiental del Distrito Federal. Este SC ocupa una extensión aproximada de 87297.1 ha, y se localiza principalmente al sur y sur poniente de la CDMX, ver: http://www.paot.org.mx/centro/programas/suelo-corena.pdf
} 
La Tabla 1, presenta la distribución de más de 600 desastres registrados en la Base de datos de DesInventar $^{5}$ en la CDMX, durante el periodo de 1970 a 2010. Se identifica que, si bien AAO no es el municipio con mayor número de desastres en la CDMX, considerando eventos geológicos e hidrometeorológicos como: hundimientos, deslizamiento e inundación por lluvias; si concentra el mayor número de ocurrencia de deslizamientos, evento asociado a PRM. Dichas condiciones físicas y sociales, que se manifiestan en una alta ocurrencia de desastres, hacen de este territorio un laboratorio para la comprensión y gestión del riesgo, considerando los factores determinantes y detonantes en los procesos de remoción en masa.

Fuente: Elaboración propia a partir del Instituto Nacional de Geografía e Informática (INEGIITER), 2010.

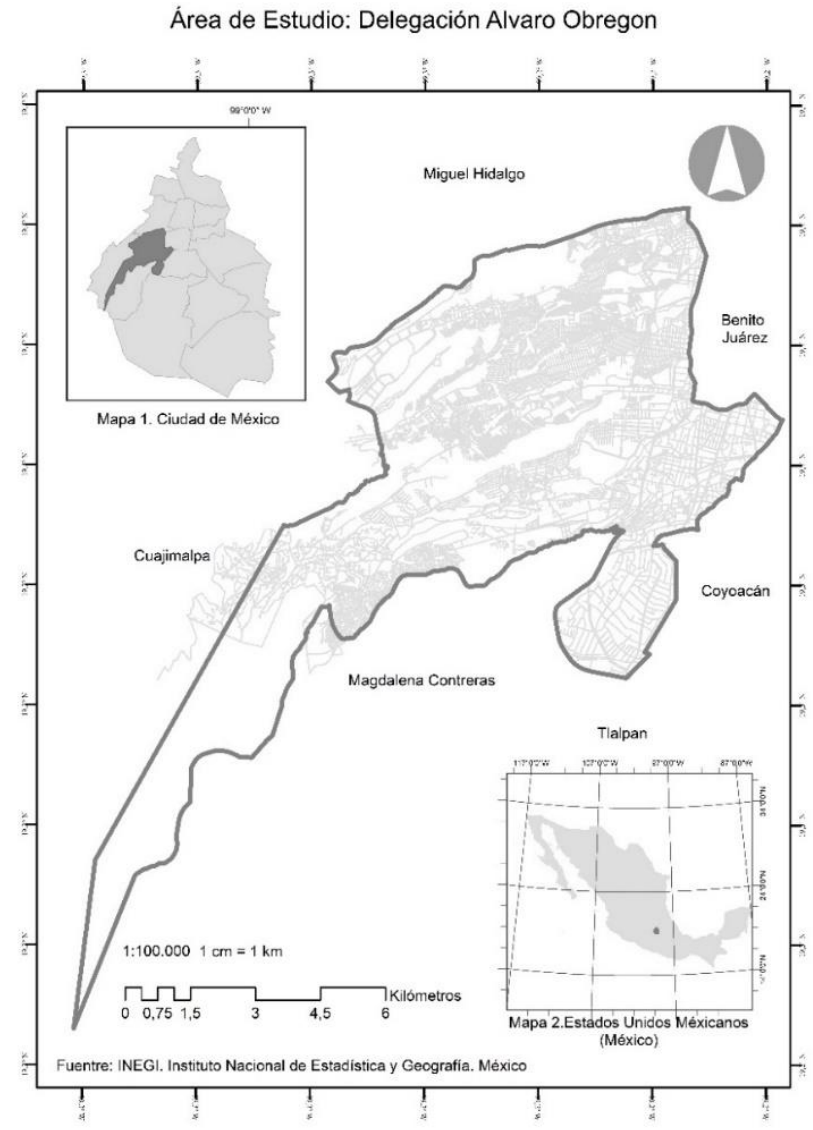

Figura 1. Localización de la alcaldía Álvaro Obregón (AAO), Ciudad de México

\footnotetext{
${ }^{5}$ DesInventar es una herramienta conceptual y metodológica para la construcción de bases de datos de pérdidas, daños o efectos ocasionados por emergencias o desastres a nivel local, particularmente desde fuente hemerográficas. Fue creado para facilitar la elaboración de estadísticas locales, y para asignar el espacio distribución de los desastres y las variaciones en la ocurrencia de desastres, ver en: https://www.desinventar.org.. Los datos presentados corresponden a la investigación doctoral de la autora, resultados aún no publicados.
} 
Tabla 1. Distribución de desastres asociados a eventos geológicos e hidrometeorológicos en CDMX, 1970 a 2010.

\begin{tabular}{|l|c|c|c|c|}
\hline \multicolumn{1}{|c|}{ Municipio } & Hundimiento & Deslizamiento & Lluvia e Inundación & Total \\
\hline Álvaro Obregón & 1 & 34 & 15 & $\mathbf{5 0}$ \\
\hline Azcapotzalco & 2 & & 18 & $\mathbf{2 0}$ \\
\hline Benito Juárez & 1 & 1 & 37 & $\mathbf{3 9}$ \\
\hline Coyoacán & 3 & & 29 & $\mathbf{3 2}$ \\
\hline Cuajimalpa & & 24 & 20 & $\mathbf{4 4}$ \\
\hline Cuauhtémoc & 3 & 1 & 71 & $\mathbf{7 5}$ \\
\hline Gustavo A. Madero & 3 & 14 & 56 & $\mathbf{7 3}$ \\
\hline Iztacalco & 1 & & 16 & $\mathbf{1 7}$ \\
\hline Iztapalapa & 16 & 1 & 76 & $\mathbf{9 3}$ \\
\hline La Magdalena C. & & 14 & 25 & $\mathbf{3 9}$ \\
\hline Miguel Hidalgo & 1 & 7 & 52 & $\mathbf{6 0}$ \\
\hline Milpa Alta & & 2 & 8 & $\mathbf{1 0}$ \\
\hline Tláhuac & 1 & 2 & 20 & $\mathbf{2 3}$ \\
\hline Tlalpan & & 7 & 37 & $\mathbf{4 4}$ \\
\hline Venustiano Carranza & 1 & 1 & 33 & $\mathbf{3 5}$ \\
\hline Xochimilco & 2 & 2 & 28 & $\mathbf{3 2}$ \\
\hline CDMX Total & $\mathbf{3 5}$ & $\mathbf{1 1 0}$ & $\mathbf{5 4 1}$ & $\mathbf{6 8 6}$ \\
\hline
\end{tabular}

Fuente: Elaboración propia, a partir de la Base de datos de Des Inventar 1970 a 2010.

\section{Métodos y materiales}

Para determinar la susceptibilidad a PRM se realizó un análisis secundario de datos, bajo el método heurístico en combinación de elementos del Proceso Analítico Jerárquico-AHP, por sus siglas en inglés (Saaty, 1977), en el que los autores asignaron categorías a los factores causales, de acuerdo a su experiencia (Kaur, Gupta, Parkash, y Thapa, 2018; Paz Tenorio et al., 2017). Esta evaluación mixta de la susceptibilidad de PRM, se procesó usando la herramienta de "suma de rásteres", la cual agrega (suma) los valores de dos rásteres celda por celda, de la extensión de Spatyal Analyst, a través del Sistema de Información Geográfica ArcGIS 10.3 y el resultado, se comparó con un inventario de datos, basado en 196 eventos de PRM, atendidos y sistematizados por la Dirección de Protección Civil y Zonas de Alto Riesgo de AAO (Protección Civil, 2011).

La edición final de los mapas, se realizó a una escala de 1:100,000, derivada de fuentes oficiales de información como: cartografía vectorial del INEGI, a escala 1:250,000; cartografía del Servicio Geológico Mexicano (SGM), a escala 1:250,000; el Sistema de Integración Territorial (INEGIITER, 2010); el conjunto de datos vectoriales de información topográfica E14A39 Ciudad de 
México, a escala 1:50,000, serie III; y dos tesis de la zona de aplicación (Cordero Estrada, 1992; Galvan García, 2012) principalmente.

Siguiendo a Vojteková y Vojtek (2020), una vez identificados los factores determinantes y detonantes con mayor probabilidad de incidir en la ocurrencia de estos eventos, se categorizan según sus atributos, con base en la literatura especializada y criterio de expertos, en este caso, en rangos de menor a mayor susceptibilidad de ocurrencia, se asignó un valor numérico, que va de uno a cinco (Tabla 2), con la finalidad de realizar una superposición de rásteres (Figura 2), así establecer los cinco niveles de susceptibilidad de "muy baja a muy alta", correspondiente a los valores de los atributos de cada capa temática (factor) y derivar seis mapas de amenaza, correspondientes a la mitad lluviosa del año, en el territorio.

A partir de la revisión de literatura, se seleccionaron siete variables (capas temáticas) para el análisis, organizados por factores determinantes como: geología del terreno, el ángulo de inclinación de las laderas (pendiente), el sistema de geoformas, los diversos usos de suelo, la energía y geometría del relieve, así como la incidencia de factores detonantes para determinar la ocurrencia de PRM, como la intensidad máxima de la precipitación por mes, en 24 horas-

Tabla 2. Matriz de factores determinantes y detonantes en los procesos de remoción en masa, clasificación de sus atributos.

\begin{tabular}{|c|c|c|c|c|c|}
\hline \multirow{2}{*}{ Factores } & \multicolumn{5}{|c|}{ Niveles de susceptibilidad } \\
\hline & Muy Baja & Baja & Media & Alta & Muy Alta \\
\hline Geología (A) & Lacustre (1) & Andesita (2) & Basalto (3) & $\begin{array}{l}\text { Aluvial } \\
(4)\end{array}$ & $\begin{array}{l}\text { Piroclástico } \\
\text { (5) }\end{array}$ \\
\hline Uso de suelo (B) & Bosque (1) & Pastizal (2) & $\begin{array}{l}\text { Área Agrícola } \\
\text { (3) }\end{array}$ & \multicolumn{2}{|c|}{ Urbanización (4) } \\
\hline Pendientes (C) & $0-6^{\circ}(1)$ & $6.1^{\circ}-12^{\circ}(2)$ & $12.1^{\circ}-18^{\circ}(3)$ & $\begin{array}{l}18.1^{\circ}-4^{\circ} \\
(4)\end{array}$ & $>=24.1^{\circ}(5)$ \\
\hline Geoformas (D) & $\begin{array}{l}\text { Pie de monte } \\
\text { inferior } y \\
\text { coladas de lava } \\
\text { (1) }\end{array}$ & $\begin{array}{l}\text { Conos } \\
\text { volcánicos } \\
\text { erosionados } \\
\text { (2) }\end{array}$ & $\begin{array}{l}\text { Laderas } \\
\text { montañosas } \\
\text { (3) }\end{array}$ & \begin{tabular}{|l|} 
Pie de \\
monte \\
superior \\
$(4)$
\end{tabular} & $\begin{array}{l}\text { Barrancas } \\
\text { (5) }\end{array}$ \\
\hline $\begin{array}{ll}\text { Energía } & \text { del } \\
\text { relieve }(E) & \end{array}$ & $0-20 \mathrm{~m}(1)$ & $20-50 \mathrm{~m} \mathrm{(2)}$ & $50-100 \mathrm{~m} \mathrm{(3)}$ & $\begin{array}{l}100-200 \\
\mathrm{~m}(4)\end{array}$ & $>200 \mathrm{~m} \mathrm{(5)}$ \\
\hline $\begin{array}{l}\text { Geometría } \\
\text { ladera }(F)\end{array}$ & Plano (1) & \multicolumn{2}{|l|}{ Cóncavo (2) } & \multicolumn{2}{|c|}{ Convexo (3) } \\
\hline Precipitación (G) & $0-5 \mathrm{~mm}(1)$ & $6-15 \mathrm{~mm}(2)$ & $16-30 \mathrm{~mm}(3)$ & $\begin{array}{l}31-50 \\
\mathrm{~mm}(4)\end{array}$ & $>51 \mathrm{~mm}(5)$ \\
\hline
\end{tabular}

Fuente: Adaptado de Ayala-Carcedo, 2002; Galvan-García, 2012; Hernández-Moreno, 2011. 
Fuente: Elaboración propia.

\begin{tabular}{|l|l|l|l|}
\hline 1 & 1 & 1 & 1 \\
\hline 1 & 5 & 3 & 1 \\
\hline 1 & 3 & 4 & 1 \\
\hline 1 & 1 & 1 & 1 \\
\hline
\end{tabular}$+$\begin{tabular}{|l|l|l|l|}
\hline 1 & 1 & 1 & 1 \\
\hline 1 & 2 & 4 & 1 \\
\hline 1 & 4 & 3 & 1 \\
\hline 1 & 1 & 1 & 1 \\
\hline
\end{tabular}$+$\begin{tabular}{|l|l|l|l|l|}
\hline 2 & 1 & 1 & 1 \\
\hline 1 & 1 & 2 & 1 \\
\hline 1 & 4 & 5 & 1 \\
\hline 1 & 1 & 1 & 1 \\
\hline
\end{tabular}$=$\begin{tabular}{|l|l|l|l|l|}
\hline 4 & 3 & 3 & 3 \\
\hline 3 & 8 & 9 & 3 \\
\hline 3 & 11 & 12 & 3 \\
\hline 3 & 3 & 3 & 3 \\
\hline
\end{tabular}

Figura 2. Sobreposición ráster multifactorial.

A continuación, se describen los insumos y el tratamiento para la obtención del índice de susceptibilidad a PRM (Figura 3).

Geología (A). Siguiendo a Alcántara y Murillo (2008), la geología como factor determinante permite establecer el tipo de roca (litología) que está involucrado en un PRM, así como su disposición estratigráfica. "Si el lecho rocoso subyacente presenta superficies por las que se pueda deslizar el material, se incrementa el potencial de ocurrencia de un movimiento. También los planos de estratificación, las fracturas, diaclasas o esquistosidad, entre otros, pueden influir en la ocurrencia de los procesos de remoción en masa" (Alcántara y Murillo, 2008, p. 53). Para este índice se incluyó la litología derivada de la cartografía existente en la Carta Geológica-Minera de la Ciudad de México E14-2, Hidalgo, Estado de México, Morelos, Puebla, Tlaxcala, del Servicio Geológico Mexicano (SGM-2002), a escala 1:250,000, se obtuvieron cinco unidades litológicas correspondiente a piroclastos, basalto, andesita, aluvial, y lacustre (Figura 3A).

Uso de suelo (B). La variabilidad en los diferentes usos de suelo y coberturas del terreno en estrecha relación con la litología, influyen notablemente en la infiltración del agua hacia el suelo (Muñoz-Iniestra, Ferreira, Escalante, López, 2013). Galván García (2012) señala que, en un contexto geomorfológico, la existencia de vegetación debe ser interpretada con un carácter dual: de protección (mitigación del efecto erosivo) o catalizador de procesos de degradación fluvial y gravitacional, dado el vínculo asociado entre la cubierta vegetal, el relieve y los agentes modeladores. Para AOO, se obtuvo una zonificación de cuatro áreas, donde el uso urbano es el predominante, seguido por un área agrícola, de pastizal y de bosque (Figura 3B), lo anterior fue derivado de la cartografía del conjunto de Datos Vectorial Edafológico Serie II (Continuo Nacional) y de Uso del Suelo y Vegetación Serie III, a escala 1:250 000, INEGI (2016). 
Pendiente (C). Este factor pretende clasificar el relieve a partir de su inclinación, con el fin de establecer la relación entre la dinámica de las laderas, la morfología y la estructura geológica (Galvan García, 2012). La inclinación del terreno se vincula con la disminución o aumento de la influencia de la fuerza gravitatoria sobre los diversos materiales y, por consecuencia, en su estabilidad o caída (Alcántara y Murillo, 2008). A partir del conjunto de datos vectoriales de información topográfica de la hoja cartográfica E14A39 (Ciudad de México), a escala 1:50,000 serie III, INEGI (2010), se obtuvo una clasificación de pendientes, a partir de los valores propios de inclinación del terreno en la AAO, en cinco niveles de gradiente (Figura 3C).

Unidades Geomorfológicas (D). Otro de los factores que influye notablemente en la probabilidad de ocurrencia de los PRM, es la propia configuración del territorio, en geoformas como resultado de la interacción de procesos endógenos y exógenos (Alcántara y Murillo, 2008 y Galvan-García, 2012). A partir de la información geológica de AOO, obtenida de la Carta Geológica-Minera Ciudad de México E14-2 (SGM, 2002), y junto con los trabajos de Cordero-Estrada (1992); LugoHubp et al., (1995); y Galván-García (2012), se realizó una zonificación de las geoformas más relevantes del área de estudio, entre ellas: barrancas, piedemonte superior, laderas montañosas, conos volcánicos erosionados, pie de monte inferior y coladas de lava (Figura 3D).

Energía del Relieve (E). Es un factor integrador de la disección en planta y la disección vertical, junto a la pendiente (Díaz, 2002, en Galván García, 2012, p. 73). Siendo un indicador comparativo entre la actividad endógena y exógena, que expresa la potencialidad del terreno a magnificar los procesos morfodinámicos (fluviales y gravitacionales). Los datos se obtienen de restar a la altitud máxima, la mínima en un área de $1 \mathrm{~km}^{2}$; los valores altos indican la posibilidad de modificaciones a la morfología original por fuerzas exógenas de gran dinámica y los valores mínimos, representarían zonas con aparente estabilidad. Este índice morfométrico, por sí mismo, no es suficiente para explicar la ocurrencia de procesos gravitacionales, por lo que se hace necesaria su utilización en combinación con otros factores como la litología, el ángulo de inclinación de las pendientes, el tipo de suelo y cobertura, etc. A partir de la información de AOO, ya obtenida, se realizó el proceso descrito y se obtuvo una zonificación de la energía del relieve, con valores del 0 a más de 200 metros (m), que se clasificaron en cinco niveles: 1) Muy Baja de 0-20 m; 2) Baja de 20-50 m; 3) Media de 50-100 m; 4) Alta de 100-200 m y 5) Muy Alta > 200 m (Figura 3E). 
Geometría del terreno (F). Es otro factor de importancia, ya que la curvatura de las superficies influye en el ángulo de reposo de los materiales y, por consiguiente, en su grado de estabilidad con respecto a la pendiente (Alcántara y Murillo, 2008 y Galvan García, 2012). La geometría influye en la dirección y comportamiento de los escurrimientos por la forma de la pendiente, es decir, la concentración o dispersión del escurrimiento depende de la morfología de ésta, sea convexa, cóncava o recta (Hernández Mena, 2008). Ésta se obtiene, calculando la derivada segunda de la superficie, a partir de un modelo digital de elevación (DEM), usando una digitalización de cartografía topográfica, con una resolución de 50 a100 metros, por medio de la función de curvatura (slope) de la extensión de análisis espacial en ArcGis. En lo general y para el caso de AAO, una curvatura positiva indica que la superficie es convexa hacia arriba en esa celda, una curvatura negativa indica que la superficie es cóncava y un valor 0 indica que la superficie es plana (Figura $3 \mathrm{~F})$.

La Precipitación (G). Es el factor detonante que más está involucrado en los procesos de remoción en masa, por los efectos que ésta tiene en el suelo, es decir la saturación del terreno y la pérdida de resistencia y cohesión (Hernández Moreno, 2011). Siguiendo a Alcántara (2008, p. 52), el régimen de precipitación en una zona se refiere a la cantidad de lluvia que cae, en promedio, en un año, el dato numérico se expresa en milímetros anuales y se toma de la estación meteorológica más cercana al evento; estos datos se pueden recuperar de las cartas hidrológicas o de las estaciones meteorológicas. En AAO, este factor se vuelve imprescindible para su estudio, ya que las características geológico-estructurales y geomorfológico-morfométricas también se vuelven complejas generando una elevada heterogeneidad de los procesos dinámicos de este tipo ( LugoHubp et al., 1995; Galvan-García, 2012).

Para la elaboración de este Índice de amenaza por PRM, se determinó el promedio de intensidad máxima mensual de la lluvia en 24 horas (Magaña, Pérez y Méndez, 2003; Ramirez Cruz, López e Ibañez, 2015; Herrera, Magaña y Morett, 2018), durante la mitad lluviosa del año, del mes de mayo a octubre de una serie de tiempo entre 1988 y 2009, a partir de los registros del Servicio Meteorológico Nacional (SMN) ${ }^{6}$. La información de precipitación diaria se extrajo de 20 estaciones

\footnotetext{
${ }^{6}$ El Servicio Meteorológico Nacional (SMN) es el organismo encargado de proporcionar información sobre el estado del tiempo a escala nacional y local en México. El SMN, depende de la Comisión Nacional del Agua (CONAGUA), la cual forma parte de la Secretaría de Medio Ambiente y Recursos Naturales (SEMARNAT), consultado en: https://smn.conagua.gob.mx/es/
} 
meteorológicas de este municipio y cinco circundantes como: Magdalena Contreras, Benito Juárez, Miguel Hidalgo, Cuajimalpa y Ocoyoacac (Estado de México). Con estos promedios, se estableció la categorización en rangos, con base en la "Red de Alerta Temprana Meteorológica de Sacmex $\mathrm{CDMX}^{\prime \prime}$, con lo que se elaboró un nivel de alerta local por lluvias (Tabla 3).

Fuente: Elaboración propia con base a información cartográfica de INEGI $(1998,2010)$, INEGI, CONABIO (2016) y del SGM (2002).
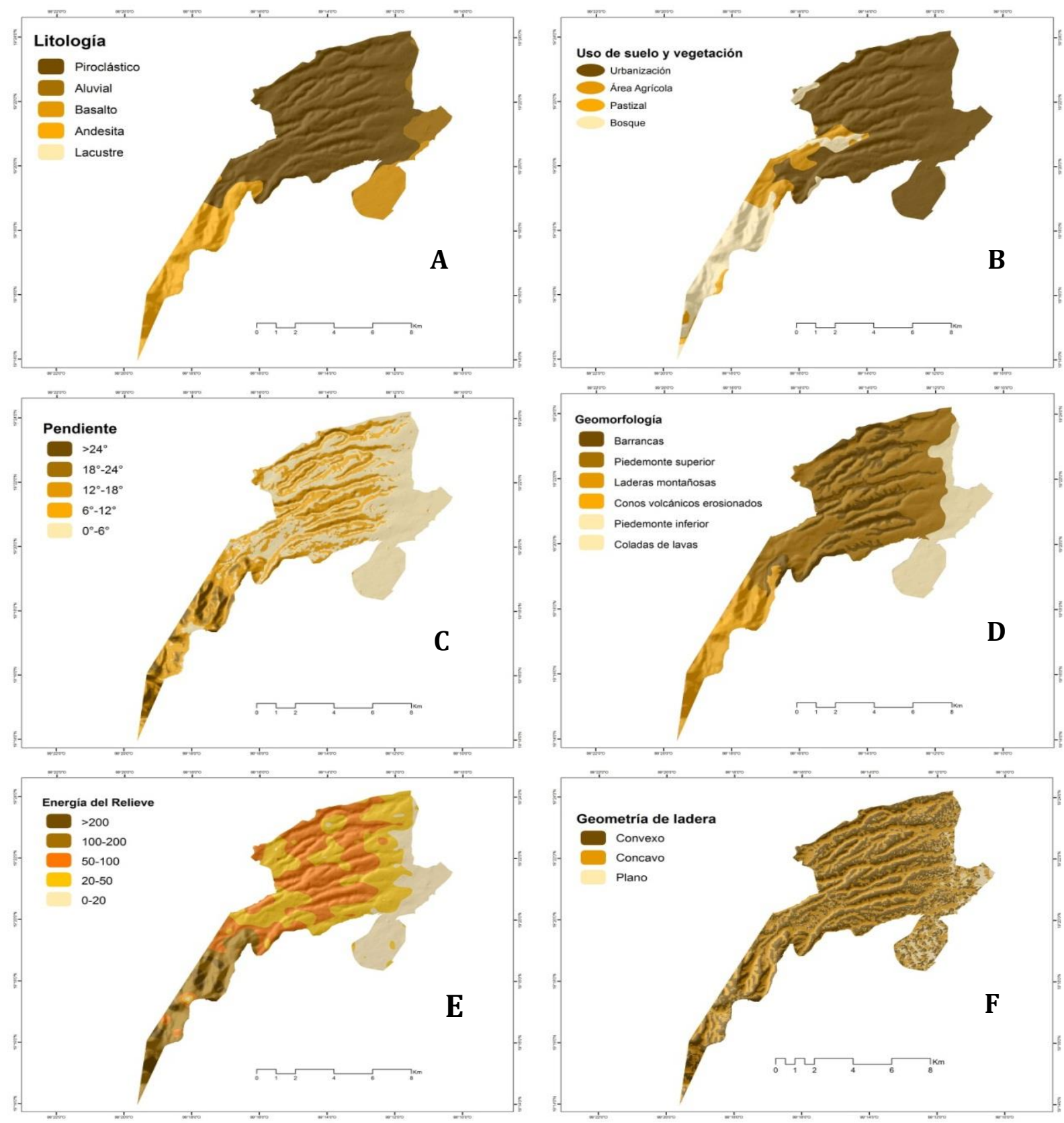

Figura 3. Capas (variables) obtenidas de los factores determinantes a PRM.

\footnotetext{
${ }^{7}$ Adaptado de la clasificación de los niveles de alerta por fenómenos hidrometeorológicos en la CDMX, dentro de la Alerta Temprana Meteorológica, de la Secretaría de Protección Civil. Ver en: https://www.archivo.cdmx.gob.mx/vivecdmx/post/alerta-temprana-meteorologica-cdmx
} 
Tabla 3. Promedio anual de número de días con lluvias, según niveles de amenaza, poniente de la CDMX, 1988-2009 (mayo-octubre).

\begin{tabular}{|c|c|c|c|c|c|c|}
\hline \multicolumn{7}{|c|}{ Niveles de intensidad de lluvia en $\mathbf{2 4}$ horas (mm) } \\
\hline $\mathbf{N}^{\circ}$ & Estaciones & $\begin{array}{l}0 \text { a } 5(\mathrm{~mm}) \\
\text { Muy baja/ } \\
\text { o ligera }\end{array}$ & $\begin{array}{l}6 \text { a } 15 \quad(\mathrm{~mm}) \\
\text { Baja/Ligera- } \\
\text { Regular }\end{array}$ & $\begin{array}{l}16 \quad \text { a } \quad 30(\mathrm{~mm}) \\
\text { Media/Regular- } \\
\text { Fuerte }\end{array}$ & $\begin{array}{|lrrr|}31 & \text { a } & 50 & (\mathrm{~mm}) \\
\text { Alta/ } & \text { Lluvias } \\
\text { fuertes } & \text { a } \\
\text { tormentas } & \\
\end{array}$ & \begin{tabular}{|lr}
$50 \quad(\mathrm{~mm})$ & Muy \\
Alta/ Tormenta \\
intensa & Tr \\
torrencial & \\
\end{tabular} \\
\hline 1 & Centro Universidad & 23.2 & 4.72 & 2.48 & 0.15 & 0.12 \\
\hline 2 & Del Valle & 25.89 & 3.09 & 1.57 & 0.05 & 0.09 \\
\hline 3 & $\begin{array}{l}\begin{array}{l}\text { Desierto de los } \\
\text { Leones }\end{array} \\
\end{array}$ & 21.34 & 5.77 & 3.18 & 0.23 & 0.16 \\
\hline 4 & Tanque el Cartero & 22.74 & 5.01 & 2.64 & 0.16 & 0.13 \\
\hline 5 & El Zarco & 21.97 & 5.45 & 2.96 & 0.15 & 0.14 \\
\hline 6 & Huayatla & 23.11 & 4.76 & 2.51 & 0.15 & 0.12 \\
\hline 7 & La Venta & 21.9 & 5.47 & 2.95 & 0.2 & 0.15 \\
\hline 8 & Monte Alegre & 21.77 & 5.82 & 2.83 & 0.17 & 0.09 \\
\hline 9 & Planta Chapultepec & 24.61 & 3.92 & 1.95 & 0.08 & 0.13 \\
\hline 10 & Radiocomunicación & 24.98 & 3.71 & 1.78 & 0.15 & 0.05 \\
\hline 11 & Río Magdalena & 21.61 & 5.52 & 3.19 & 0.13 & 0.23 \\
\hline 12 & $\begin{array}{ll}\text { San } & \text { Bartolo } \\
\text { Ameyalco } & \\
\end{array}$ & 22.84 & 4.7 & 2.8 & 0.21 & 0.12 \\
\hline 13 & Tanque San Joaquín & 23.55 & 4.46 & 2.39 & 0.15 & 0.12 \\
\hline 14 & San Francisco & 23.02 & 4.79 & 2.59 & 0.15 & 0.13 \\
\hline 15 & Santa Fé & 23.15 & 4.38 & 2.77 & 0.23 & 0.15 \\
\hline 16 & Tanque Lienzo & 23.37 & 4.66 & 2.36 & 0.14 & 0.14 \\
\hline 17 & Tanque Santa Lucía & 23.33 & 4.54 & 2.54 & 0.15 & 0.13 \\
\hline 18 & Tarango & 24.45 & 4.43 & 1.68 & 0.07 & 0.05 \\
\hline 19 & Triángulo & 23.87 & 4.24 & 2.32 & 0.12 & 0.13 \\
\hline 20 & Yaqui & 22.05 & 5.27 & 2.89 & 0.27 & 0.2 \\
\hline
\end{tabular}

Fuente: Elaboración propia con base al registro del Servicio Meteorológico Nacional (SMN).

A partir de estos datos, se realizó un mapa isoyético medio mensual de 21 años, que se calibró a través de métodos de interpolación para el trazo de isolíneas, por medio del método Spline con la herramienta Kriging de la extensión Spatial Analyst, con lo que se obtuvo seis mapas de la intensidad máxima mensual de lluvia en la AAO, determinando las zonas de mayor precipitación y el mes correspondiente, como se observa en la (Figura 4). 
Fuente: Elaboración propia.

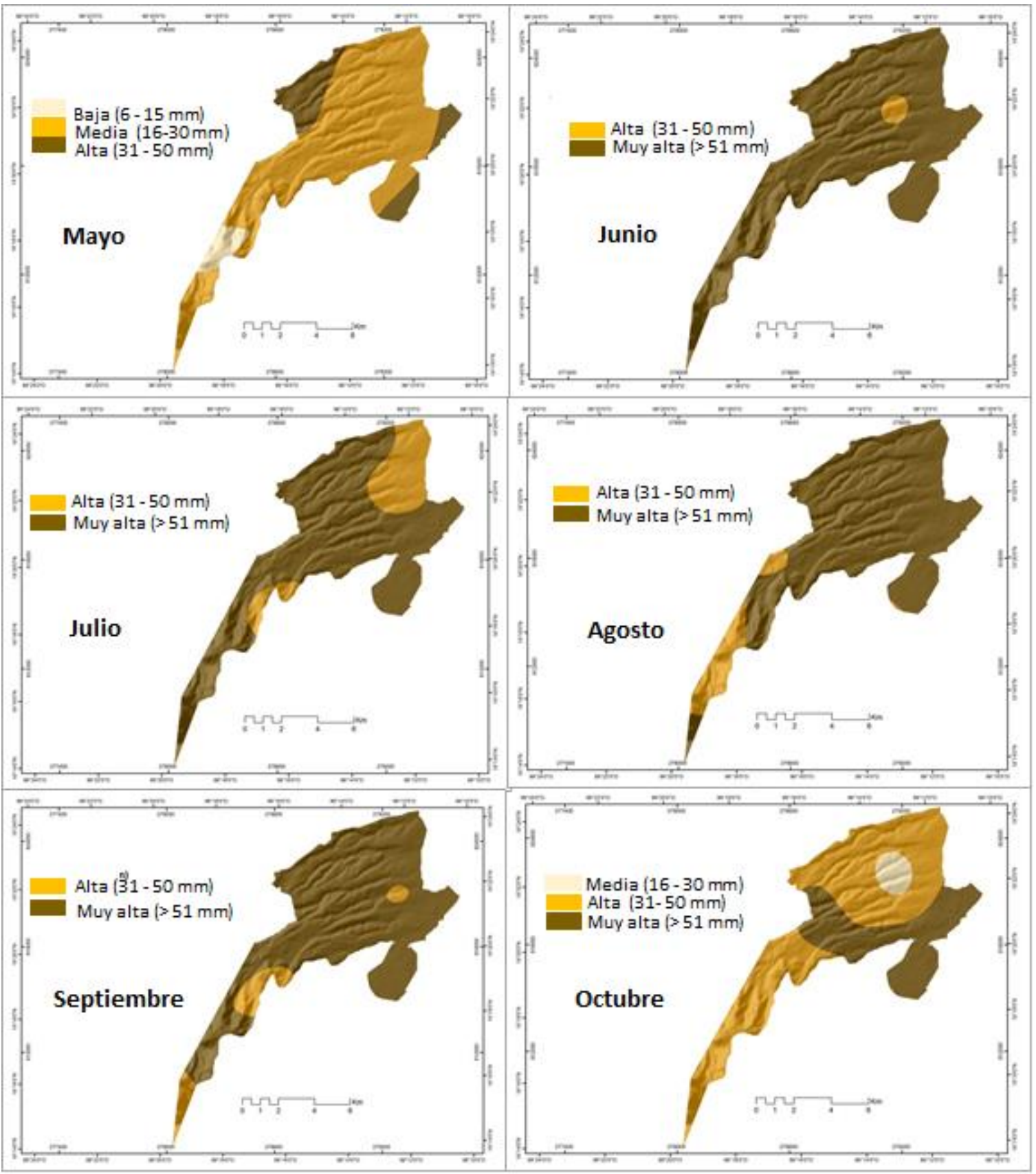

Figura 4. Promedio de la intensidad máxima mensual de precipitación en 24 horas, alcaldía de Álvaro Obregón, 1988 a 2009.

Mapa índice de amenaza. Como ya se mencionó, este índice es una zonificación de áreas susceptibles a PRM, que resultó de sobreponer los índices cualitativos de la distribución de cada variable en su representación cartográfica, en un nuevo documento, a través de ArcGIS, presentando seis momentos, según el comportamiento máximo mensual de lluvias. Para corroborar 
estos resultados, se sobrepuso el registro de 196 eventos de PRM, atendidos por Protección Civil, durante el periodo de 2007 a 2010, tipo inventario de la Dirección de Protección Civil y Zonas de Alto Riesgo de AAO (2011), con lo que se validó el resultado de este índice, lo estimado con respecto a lo reportado (inventario), mostrando una dinámica cercana a la realidad.

\section{Resultados}

La metodología aplicada proporcionó, como resultado, un modelo de zonificación de la amenaza por procesos de remoción en masa, en la Alcaldía Álvaro Obregón, considerando el periodo de lluvias que va de mayo a octubre (Figura 5). Con este índice, se determinó que alrededor del $85 \%$ del territorio de la alcaldía, presenta susceptibilidad mediana a muy alta; en promedio el $60 \%$ de su territorio presenta una amenaza de alta a muy alta, (Tabla 4) siendo los meses de junio a septiembre los más críticos, particularmente el mes de julio (Figura 5A). En general, estos resultados indican la alta inestabilidad de las laderas de AAO. Este resultado se constató con el registro de más de 190 eventos atendidos por Protección Civil de esta demarcación.

Tabla 4. Evaluación de la amenaza por PRM en los periodos de lluvia 1988 a 2009 en AAO.

\begin{tabular}{|l|l|l|l|l|l|l|l|l|l|l|l|}
\hline \multirow{2}{*}{ Mes } & \multicolumn{2}{|l|}{ Muy Baja } & \multicolumn{2}{l|}{ Baja } & \multicolumn{2}{l|}{ Media } & \multicolumn{2}{l|}{ Alta } & \multicolumn{2}{l|}{ Muy Alta } \\
\cline { 2 - 12 } & Área (Ha) & \% & Área (Ha) & \% & Área (Ha) & \% & Área (Ha) & $\%$ & Área (Ha) & \% \\
\hline Mayo & 150 & 1.9 & 1,701 & 21.2 & 3,099 & 38.5 & 2,225 & 27.7 & 869 & 10.8 \\
\hline Junio & 5 & 0.1 & 1,025 & 12.7 & 1,704 & 21.2 & 2,420 & 30.1 & 2,891 & 35.9 \\
\hline Julio & 5 & 0.1 & 1,112 & 13.8 & 1,852 & 23.0 & 2,348 & 29.2 & 2,728 & 33.9 \\
\hline Agosto & 12 & 0.2 & 1,099 & 13.7 & 1,723 & 21.4 & 2,284 & 28.4 & 2,926 & 36.4 \\
\hline Septiembre & 5 & 0.1 & 1,066 & 13.3 & 1,771 & 22.0 & 2,322 & 28.9 & 2,882 & 35.8 \\
\hline Octubre & 12 & 0.2 & 1,209 & 15.0 & 2,262 & 28.1 & 2,716 & 33.8 & 1,845 & 22.9 \\
\hline
\end{tabular}

Fuente: Elaboración propia.

Determinar la susceptibilidad a PRM por meses lluviosos del año, permite evaluar la amenaza en un escenario temporal; como se muestra en la tabla anterior, la distribución de la susceptibilidad "Muy Baja" a PRM, presenta una superficie máxima de 150 ha, equivalentes al 1.9\% de la superficie total de la AAO en el mes de mayo, mientras que, para octubre, ésta se reduce a 12 ha, equivalentes al $0.2 \%$. Esto concuerda con el mes de menor precipitación intensa, que oscila de los 6 hasta los $50 \mathrm{~mm}$ (6 a 50 lts $/ \mathrm{m}^{2}$ ), en la unidad de análisis, al inicio de la temporada lluviosa y termina con precipitaciones que van de los 16 hasta más de $50 \mathrm{~mm}\left(16\right.$ a $>50$ lts $\left./ \mathrm{m}^{2}\right)$, de acuerdo a la Tabla 3. 
La "Baja" y "Media" amenaza por PRM en AAO, representan el mismo patrón que el anterior, a excepción del tiempo, que va de agosto a septiembre, pues aquí la probabilidad "Baja" disminuye ligeramente (13.7-13.3\%), mientras que en la "Media" aumenta de manera ligera (21.4-22\%). La "Alta" amenaza, presenta una tendencia relativamente ascendente durante la temporada de lluvias, que va de $27.7 \%$ en mayo, a $33.8 \%$ en octubre. Durante los meses comprendidos entre junio a agosto, presenta un ligero decremento que varía de $30.1 \%$ a $29.2 \%$ y $28.4 \%$, respectivamente.

Finalmente, la amenaza de ocurrencia de procesos de remoción en masa clasificada como "Muy Alta", es menor para principios de la temporada de lluvias (mayo), distribuyéndose por 869 ha, equivalentes al $10.8 \%$ de la superficie. Ésta se eleva notablemente para el periodo comprendido entre junio y septiembre, con una tendencia estable, y una media de 2,857 ha, equivalentes al 36\% de la superficie de la AAO. Por último, para el mes de octubre, esta probabilidad baja considerablemente, en unas 1,845 ha, equivalentes al $23 \%$ de la superficie. 
Fuente: Elaboración propia.

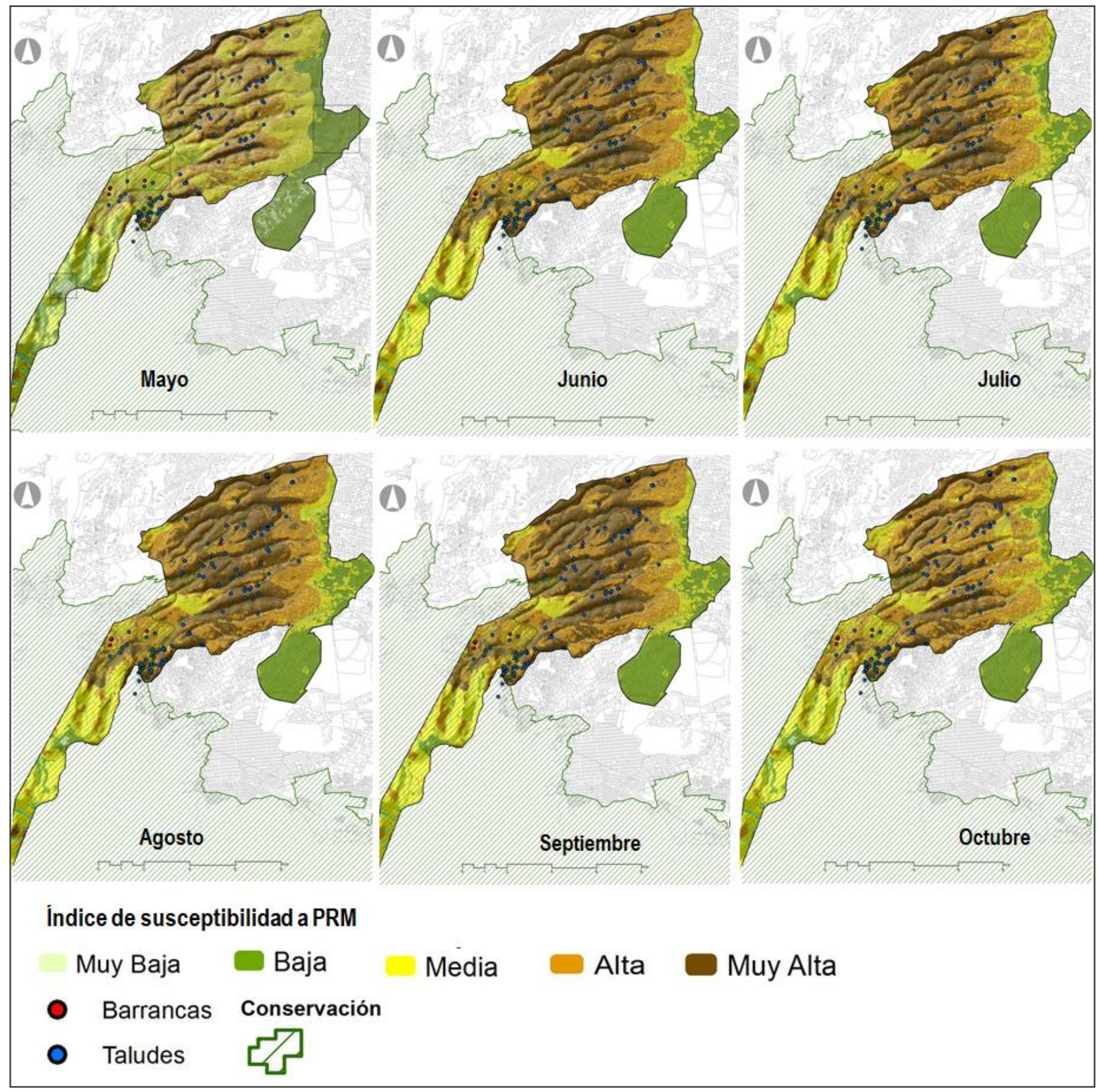

Figura 5. Índice de Amenaza por PRM en AAO, correspondiente a los seis meses lluviosos del año. 
Fuente: Elaboración propia.

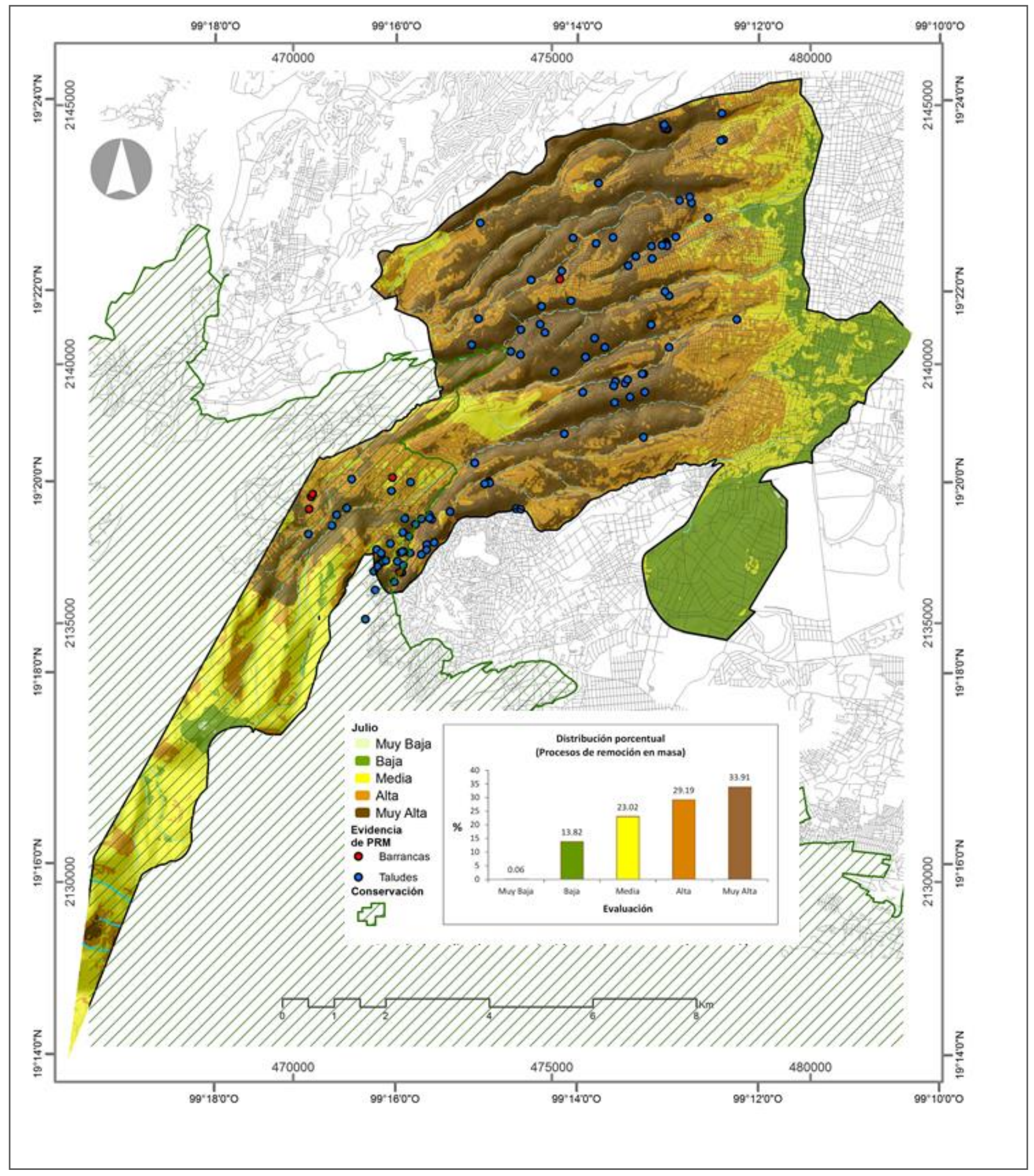

Figura 5a. Índice de Amenaza por PRM en AAO, mes de julio 


\section{Discusión}

Se propuso y aplicó la susceptibilidad a procesos de remoción en masa (PRM) como un elemento para la evaluación de la amenaza, a partir del método heurístico, en combinación de siete factores: seis determinantes (geología, uso de suelo y edafología, pendiente, geoforma, energía del relieve y geometría de la ladera), y un factor detonante de la amenaza (precipitación), con base en una revisión documental, el conocimiento en campo de la alcaldía Álvaro Obregón, en la Ciudad de México, y criterio de expertos, con lo que se determinaron cinco niveles de amenaza por PRM en este territorio. A través de una suma ponderada de rásteres con ayuda de un SIG, se obtuvo su proporción en hectáreas y los momentos (meses) de mayor propensión a la ocurrencia de estos eventos, dado la presencia de lluvias intensas (Tabla 4 y Figura 5).

Los resultados de este índice aplicado en AAO, en alguna medida, corresponden a los estudios realizados en la misma zona, al indicar la alta susceptibilidad a PRM, dadas las condiciones físicas del territorio e interacción con factores detonantes como la actividad antrópica, la construcción de viviendas en las barrancas y pendientes, la explotación minera que predominó en la zona, el crecimiento no controlado y los escurrimientos provocados por la precipitación (Cordero Estrada, 1992; Lugo-Hubp et al., 1995; Galván García, 2012; Atlas de Riesgo de AAO, 2014; González Rivera, 2018).

Galván García (2012, pp. 115-116) realizó un mapa de susceptibilidad a PRM, donde determinó cinco niveles de amenaza por medio de una sobreposición de índices numéricos máximos de cada mapa morfométrico (altimetría, inclinación del terreno, densidad y profundidad de la disección, energía del relieve), que posteriormente cruzó con un mapa geomorfológico para la obtención de esta zonificación. En ese resultado se establece que el 58\% del territorio de la delegación se encuentra bajo una "estabilidad aparente", solo el 13\% está frente a una "máxima ocurrencia", un $18 \%$ se encuentra en un "área potencial" a PRM, un 8.5\% "baja susceptibilidad de ocurrencia" y $2.5 \%$ en "zona excepcional".

La categoría "estabilidad aparente", en la que cae más del 50\% del AAO, sugiere ser una zona gris en la que hay incertidumbre en cuanto a la valoración o identificación de la susceptibilidad, dado que la autora señala que "en estos territorios las condiciones morfológicas y estructurales para la 
ocurrencia de procesos de ladera, no son claras o están disimuladas por la cubierta urbana de la creciente CDMX" (Galvan García, 2012, p., 77).

Esta interpretación se refuerza con lo señalado en un estudio de Quesada Román y Zamorano (2019), quienes señalan que los terrenos donde no coincidió ningún índice morfométrico en una zonificación de procesos de ladera e inundaciones, son casos aislados y en esta fase del análisis, fueron considerados como zonas de "estabilidad aparente" en el análisis realizó en la cuenca alta del río General, Costa Rica. En ese sentido y con los resultados de Galván (2012), solo un poco más del $30 \%$ del territorio es susceptible a PRM, lo que podría explicarse por una subestimación de sus resultados derivada del método utilizado.

En el Atlas de Riesgo de la Ciudad de México y en la actualización de los mapas de riesgo de Álvaro Obregón (Secretaria de Protección Civil y UNAM, 2014), se realizó un análisis de la susceptibilidad por inestabilidad de laderas, a partir de la sobreposición de cuatro variables: energía del relieve, potencial erosivo, densidad de corrientes y grado de la pendiente, por medio de una clasificación e interpolación de la información tratada por ArcGis (10.1); se representaron espacialmente los niveles de propensión a PRM. Con este y otros insumos, escalaron a la realizaron mapas de peligros, vulnerabilidad y riesgos por fenómenos de remoción en masa, donde se identifica, de manera espacial, tres niveles de amenaza por inestabilidad de laderas clasificados en alta, media y baja, presentan la ubicación de sitios en los que hubo un antecedente o evento por PRM.

Ambos resultados son informativos del grado de amenaza que existe en esta Alcaldía. El presente trabajo adiciona e informa de manera mixta (cualitativa y cuantitativamente), la amenaza por PRM; cuantifica mensualmente la porción territorial por niveles de amenaza, considerando la dimensión temporal, los patrones y variaciones espaciales de PRM, concatenados por las lluvias, factor detonante que no fue incluido por las propuestas anteriores.

Como ya se mencionó anteriormente, este tipo de metodologías, heurísticas y otras, en combinación con un análisis multicriterio, bajo la normalización de los datos, han sido replicadas y ajustadas por otros autores a nivel nacional mexicano. De estos trabajos, cabe destacar los aportes de Paz Tenorio et al. (2017), quienes realizaron un ajuste metodológico (normalización) y consistencia al incorporar el análisis multicriterio, para ajustar un mapa de susceptibilidad a procesos de remoción 
en masa, en Tuxtla Gutiérrez, Chiapas. Su calibración, entre 2012 y 2014, mostró un buen grado de confiabilidad respecto a su primer resultado.

Aunque lo anterior, sugiere la importancia de ajustar y evaluar la calidad del índice propuesto para AAO, con un método estadístico, como lo señala los trabajos anteriormente citados y una revisión sistemática de literatura internacional sobre modelos de susceptibilidad y amenazas por deslizamientos, basados en enfoques estadísticos (Reichenbach et al., 2018); también, los resultados de Paz Tenorio et al. (2017) evidencian el éxito del modelo, basado en el método heurístico, el cual se basa principalmente en el conocimiento de experto (Yilmaz y Yildirim 2006; Isik Yilmaz 2009, en Ahmed, 2015).

Esto también es demostrado en estudios internacionales como el de Kaur y colaboradores, (2018), quienes usaron idéntico método para estimar la susceptibilidad de PRM, en el estado de Sikkim, al norte de la India, a través de una superposición ponderada, asignando pesos a varios factores desencadenantes (geología/litología, morfometría de pendiente, densidad de línea, régimen de agua, lluvia, elevación, tipo de suelo, licuefacción del suelo, espesor del suelo, densidad del edificio, alivio relativo y uso de la tierra/cobertura del suelo), a través de la opinión de expertos, el modelo fue validado con un deslizamiento en el pasado, con el que se cuantificó una precisión por arriba del $80 \%$.

Aunque existen diferentes enfoques para la evaluación de la susceptibilidad o amenaza por PRM, ya sea por medio de métodos heurísticos, determinísticos o estadísticos, cada uno con sus propios alcances y limitaciones (Ciurleo, Cascini y Calvello, 2017; Reichenbach et al., 2018; Shano et al., 2020); los geógrafos y otros analistas espaciales están en la constante búsqueda de analizar, evaluar, cartografiar y sintetizar diferentes datos, de manera más efectiva, para elaborar representaciones espaciales de la susceptibilidad y la amenaza, reduciendo la complejidad para la interpretación y sobre todo la acción de los decisores en la planificación y mitigación de los riesgos derivados por estos eventos. A su vez, mancomunadamente con el desarrollo y avance de las tecnologías geoespaciales, entre ellas: sistemas de información geográfica, teledetección o percepción remota y sistemas de posicionamiento global por satélites, que facilitan este propósito (Pourghasemi et al., 2018). 


\section{Limitaciones, potencialidades y recomendaciones}

Como ya se mencionó, el alcance de este estudio es una aplicación y aproximación metodológica, de carácter mixto o semicuantitativo, para determinar la susceptibilidad o la amenaza por PRM a como base para una evaluación multicriterio de la amenaza en un territorio dado. Por tanto, no se trata de un análisis de desastres per se, ni de la elaboración de un escenario de riesgo, dado que no se abordan componentes como la vulnerabilidad, el riesgo en sí mismo, los actores y su respuesta (Riddell, van Delden, Maier, Zecchin, 2019). Este índice se realizó con información secundaria y podría ser enriquecido con otras fuentes de información, como el trabajo de campo más profundo que pretenda caracterizar y clasificar los PRM visibles en el territorio, bajo el uso de imágenes satelitales de alta resolución.

Aunque la periodicidad de la información, en cuanto al inventario de eventos de remoción de masa es de tres años (2007-2010), esta fue suficiente e incluyó 196 eventos de PRM, que requirieron de atención por parte de las autoridades locales, mismas que se lograron georreferenciar en la zona de estudio, lo que permitió validar los resultados de este índice. No obstante, con apoyo de las autoridades locales, se podría organizar un inventario siguiendo los parámetros de Alcántara y Murillo (2008) y dar continuidad temporal de este análisis, mejorando su calidad y abriendo nuevas líneas de investigación en cuanto a la evolución de los PRM.

Existe evidencia que demuestra, que la ocurrencia de PRM, provocados por la actividad humana, está aumentando, en particular en relación con la construcción, la minería ilegal y los cortes de taludes (Froude y Petley, 2018). No obstante, estos factores se incluyen de manera incipiente en los modelos o índices de susceptibilidad y amenaza, esto se podría explicar por la falta de información directa o específica sobre este tipo de detonadores (Michoud, Derron y Jaboyedof, 2011). Una revisión de literatura exhaustiva de estos factores y consulta con expertos podría agregar nuevos elementos en la valoración cualitativa y/o cuantitativa.

Una limitante relevante y recurrente en este tipo de trabajos, corresponde a la diversidad de fuentes cartográficas, dadas las variaciones en las escalas de la cartografía temática, limitante reportada en los diferentes trabajos nacionales e internacionales (Kaur et al., 2018; Paz Tenorio et al., 2017; Reichenbach et al., 2018). 
Es importante destacar, que esta metodología es una herramienta útil y sencilla, perfectible y puede replicarse de manera fácil en otro territorio, a bajo costo y con alta resolución, contando con los insumos y conocimientos necesarios; generando salidas fácilmente legibles para un público no experto, tomadores de decisiones, formuladores de políticas públicas y la comunidad, en general, como parte del fortalecimiento de sus capacidades. Todo lo anterior, en consonancia con las “direcciones futuras del $5^{\circ}$ Foro Mundial de Deslizamientos, Kyoto-2020" del Consorcio Internacional sobre Deslizamientos de Tierra (Alcántara Ayala et al., 2017).

Como paso futuro, los autores tienen el interés de calibrar este índice a un modelo cuantitativo, fortaleciendo la calidad y análisis del inventario de PRM, en posible colaboración con las autoridades locales para integrar una serie de tiempo más larga e intentar predecir la ocurrencia espacial de estos eventos, a partir de la distribución de la densidad de deslizamientos de tierra, así como escalar este índice de susceptibilidad a una evaluación integral de la amenaza y construir un escenario de riesgo.

\section{Conclusiones}

La identificación y evaluación de la amenaza por procesos de remoción en masa (PRM) por medio de un índice, es un tema importante, porque su análisis geoespacial proporciona una herramienta útil para la planeación del territorio y la prevención de desastre; en tanto que, el conocimiento del nivel de susceptibilidad y amenaza (alta y muy alta) de las áreas propensas a eventos por PRM por parte del público en general y tomadores de decisiones, ayudará a prevenir pérdidas humanas y materiales, así como a mitigar daños ocasionados por deslizamientos o colapsos del terreno. Por lo tanto, un mapeo detallado e integrado de la combinación de los factores determinantes junto con los detonantes a PRM, en este caso, por intensidad de precipitación extrema, permite determinar el comportamiento, no solo espacial sino también temporal de la amenaza, identificando así puntos y momentos críticos, propensos, a la ocurrencia de movimientos de terreno o laderas. En el caso de la alcaldía Álvaro Obregón, en el poniente de la Ciudad de México, este índice determina una alta amenaza por PRM, dada la inestabilidad de las laderas ya que alrededor del $85 \%$ de su territorio presenta de una mediana a muy alta susceptibilidad a estos procesos geomórficos, siendo los meses de junio a septiembre lo más críticos por las intensas lluvias. Este resultado es relevante para los procesos de toma de decisiones, relacionados con el ordenamiento territorial y la gestión del riesgo. 


\section{Agradecimientos}

Los autores agradecen el apoyo financiero otorgado por CONACYT, mediante la beca de doctorado de María Alejandra Cortés Ortiz (CVU-229677). De igual manera, a José Manuel Figueroa Maheng, Antonio Pablo-Pablo, de la UNAM, y a María Juliana Carvajal Ardila de UNIVALLE- Colombia, por su apoyo técnico en la elaboración de la cartografía y valiosos comentarios, que permitieron orientar los resultados obtenidos, así como a Elda Luyando, de la UNAM, quien facilitó la base de datos de precipitación de la Ciudad de México y al Ingeniero Ignacio Azpeitia Hernández, quien proporcionó los registros de eventos en AAO, bajo la Coordinación de Zonas de Alto Riesgo en AO 2000 a 2018. Finalmente, se reconoce la labor de los revisores anónimos de este manuscrito por sus observaciones y acertadas sugerencias para su mejora.

\section{Declaración de conflictos de intereses}

Los autores declaran no tener conflictos de intereses financieros ni personales que puedan influir inapropiadamente en el desarrollo de estos resultados.

\section{Contribuciones de los autores}

Este documento fue preparado utilizando las contribuciones de todos los autores. Todos los autores han leído y aprobado el manuscrito final.

\section{Referencias bibliográficas}

Abeldaño Zúñiga, R. A., y González Villoria, A. M. (2018). Desastres en México de 1900 a 2016: patrones de ocurrencia, población afectada y daños económicos. Revista Panamericana de Salud Pública, 42, 1-8. https://doi.org/10.26633/RPSP.2018.55

Ahmed, B. (2015). Landslide susceptibility mapping using multi-criteria evaluation techniques in Chittagong Metropolitan Area, Bangladesh. Landslides, 12(6), 1077-1095. https://doi.org/10.1007/s10346-014-0521-X

Alcaldía Álvaro Obregón y Protección Civil. (n.d.). Programa de Trabajo 2018-2021. Dirección de Protección Civil y Zonas de Alto Riesgo. http://www.atlas.cdmx.gob.mx/AOB/pdf/PROGRAMATRABAJO.pdf

Alcaldía Álvaro Obregón y Protección Civil-Coordinación de Zonas de Alto Riesgo (CZAR) de AAO, 2011, Registro de eventos atendidos por CZAR del 2007 a 2010 [Base de datos]. Datos Administrativos, no publicados y recuperados para investigación. CMDX. 
Alcántara Ayala, I. (2000). Landslides: ¿deslizamientos o movimientos del terreno? Definicion, clasificaciones y terminologia. Investigaciones Geográficas, 1(41). https://doi.org/10.14350/rig.59101

Alcántara Ayala I. (2010). Derrumbes: una luz al final del túnel. http://www.dgdc.unam.mx/assets/cienciaboleto/cb_13.pdf

Alcántara-Ayala, I. y Murillo-García, F.G. (2008). Mass movement processes in Mexico: towards a proposal to construct a nation-wide inventory [Procesos de remoción en masa en México: Hacia una propuesta de elaboración de un inventario nacional]. Investigaciones Geograficas, 66, 47-64. https://bit.ly/3wJScXH

Alcántara Ayala, I., Sassa, K., Mikoš, M., et al,. (2017). The 4th World Landslide Forum: Landslide Research and Risk Reduction for Advancing the Culture of Living with Natural Hazards. International Journal of Disaster Risk Science, 8(4), 498-502. https://doi.org/10.1007/s13753-017-0139-4

Aristizábal, E., Martínez, H., \& Vélez, J. I. (2010). Una revisión sobre el estudio de movimientos en masa detonados por lluvias. Revista de La Academia Colombiana de Ciencias, 34(53), 209-227.

Ayala-Carcedo, F. (2002). Análisis de riesgos por movimientos de ladera. In J. O. C. (coord. Francisco Javier Ayala Carcedo (coord.) (Ed.), Riesgos naturales (pp. 379-410). https://dialnet.unirioja.es/servlet/libro?codigo $=4251$

Borja Baeza, R.C., y Alcántara, I. (2004). Procesos de remocion en masa y riesgos asociados en zacapoaxtla, puebla. Investigaciones Geograficas, 53, 7-26. http://www.scielo.org.mx/pdf/igeo/n53/n53a2.pdf

Becerra Baeza, C., y De Rurange Espinoza, J. (2018). Modelo de susceptibilidad a procesos de remociones en masa en rutas cordilleranas de Chile Central: Ruta $115 \mathrm{CH}$, Paso Pehuenche, Región del Maule. Investigaciones Geográficas, (55), 89-110. https://doi.org/10.5354/07195370.2018.50799

Ciurleo, M., Cascini, L., \& Calvello, M. (2017). A comparison of statistical and deterministic methods for shallow landslide susceptibility zoning in clayey soils. Engineering Geology, 223(December 2016), 71-81. https://doi.org/10.1016/j.enggeo.2017.04.023

Cordero Estrada, M.F. (1992). El origen y evolución del relieve en la delegación Álvaro Obregón. Universidad Nacional Autonóma de México. https://repositorio.unam.mx/contenidos/315394

Cui, Y., Cheng, D., Choi, C. E., Jin, W., Lei, Y., y Kargel, J. S. (2019). The cost of rapid and haphazard urbanization: lessons learned from the Freetown landslide disaster. Landslides, (March), 1167-1176. https://doi.org/10.1007/s10346-019-01167-x

Froude, M. J., y Petley, D. N. (2018). Global fatal landslide occurrence from 2004 to 2016. Natural Hazards and Earth System Sciences, 18(8), 2161-2181.

Galvan García, A.H. (2012). Procesos de remoción en masa en la Delegación Álvaro Obregón : peligros asociados y su cartografía (Universidad Nacional Autónoma de México). Recuperado de https://repositorio.unam.mx/contenidos/445034 
González Rivera, D. (2018). Zonas urbanas marginadas vulnerables a procesos de remoción en masa, zona norte, delegación Álvaro Obregón, Ciudad de México, 2015-2018. Revista Geográfica, 159, 89-108. https://revistasipgh.org/index.php/regeo/article/view/171

Hernández Mena, Z. (2008). Mapa de susceptibilidad a procesos de remoción en masa con base en análisis multivariado: La Región de Zapotitlá, Puebla. UNAM. https://repositorio.unam.mx/contenidos/444078

Hernández Moreno, M.G. (2011). Procesos de remoción en masa en Motozintla de Mendoza, Chiapas. Universidad Nacional Autónoma de México (UNAM). https://bit.ly/2Rstyeb

Herrera, E., Magaña, V., y Morett, S. (2018). Relación entre eventos extremos de precipitación con inundaciones. Estudio de caso: Tulancingo, Hidalgo. Nova Scientia, 10(21), 191-206. https://doi.org/10.21640/ns.v10i21.1527

Holcombe, E. A., Beesley, M. E. W., Vardanega, P. J., y Sorbie, R. (2016). Urbanisation and landslides: hazard drivers and better practices. Proceedings of the Institution of Civil Engineers - Civil Engineering, 169(3), 137-144. https://doi.org/10.1680/jcien.15.00044

Instituto Nacional de Estadística Geografía e Informática (INEGI). (2010). Censo de Población y Vivienda 2010. Principales resultados por localidad(ITER). INEGI. https://bit.ly/3uR7TL1

Instituto Nacional de Estadística Geografía e Informática (INEGI). (2016). Uso del suelo y vegetación, escala 1:250000, serie VI (continuo nacional). CONABIO. https://bit.ly/3skMHva

Instituto Nacional de Estadística y Geografía (INEGI). (1998). Carta Topográfica 1:50,000; Ciudad de México E14A39. INEGI. https://bit.ly/2QpnDGi

Instituto Nacional de Estadística y Geografía (INEGI). (2015). Encuesta Intercensal 2015. Red Nacional de Metadatos (INEGI). https://bit.ly/3gc5LcE

International Panel for Climate Change. (2019). Climate Change and Land: IPCC report. https://bit.ly/3daCcWQ

Kaur, H., Gupta, S., Parkash, S., y Thapa, R. (2018). Knowledge-driven method: a tool for landslide susceptibility zonation (LSZ). Geology, Ecology, and Landscapes, 1-15. https://doi.org/10.1080/24749508.2018.1558024

Korup, O., Densmore, A.L., y Schlunegger, F. (2010). The role of landslides in mountain range evolution. Geomorphology, $120(1-2)$, 77-90. https://doi.org/10.1016/j.geomorph.2009.09.017

Linares-Canto, R.A. (2012). Análisis geomorfológico de la DAO usando GIS. Universidad Nacional Autonóma de México. https://repositorio.unam.mx/contenidos/240492

Lugo-Hubp, J., Cordero-Estrada, M., Zamorano-Orozco, J. (1995). Relieve, litología y riesgos en la zona urbana de la Delegación Álvaro Obregón, Distrito Federal, México. Revista Mexicana de Ciencias Geológicas, Vol. $\quad 12, \quad$ 52-67. https://dialnet.unirioja.es/servlet/articulo?codigo=281794

Magaña, V., Pérez, J., y Méndez, M. (2003). Diagnosis and prognosis of extreme precipitation events in the Mexico City Basin. Geofisica Internacional, 42(2), 247-259. 
Mansilla, E. (2001). Algunas Notas Para La Reflexión A Proposito Del Terremoto De El Salvador. https://bit.ly/323YbZc

Michoud, C., Derron, M.H., Jaboyedoff, M., Nadim, F., y Leroi, E. (2011). Classification of landslide-inducing anthropogenic activities. 5th Canadian Conference on Geotechnique and Natural Hazards, 231-233.

Murillo-García, F.G., y Alcántara-Ayala I. (2015). Landslide susceptibility analysis and mapping using statistical multivariate techniques: Pahuatlán, Puebla, Mexico. In W. W. (Ed.), Recent Advances in Modeling Landslides and Debris Flows. 179-194. https://doi.org/10.1007/9783-319-11053-0_16

Muñiz-Jauregui, J. A., \& Hernández-Madrigal, V. M. (2012). Zonificación de procesos de remoción en masa en puerto vallarta, jalisco, mediante combinación de análisis multicriterio y método heurístico. Revista Mexicana de Ciencias Geologicas, 29(1), 103-114.

Muñoz Iniestra, D.J, Ferreira Ramírez, M., Escalante Arriaga, I.B., y López García, J. (2013). Relación entre la cobertura del terreno y la degradación física y biológica de un suelo aluvial en una región semiárida. Terra Latinoamericana, 31(3), 201-210. https://bit.ly/2ODX6nY

Nojavan M., y Salehi E. (2017). An analysis of the effects of human settlements on increasing and strengthening the natural disasters : a case study of Iran. Journal of Research in Ecology, 5 (1), 515-530. https://issuu.com/reviewjre/docs/ec0336

Pardeshi, S.D., Autade, S.E., y Pardeshi, S.S. (2013). Landslide hazard assessment: Recent trends and techniques. SpringerPlus, 2(1), 1-11. https://doi.org/10.1186/2193-1801-2-523

Paz Tenorio, J.A., González Herrera, R., Gómez Ramírez, M., y Velasco Herrera, J.A. (2017). Metodología para elaborar mapas de susceptibilidad a procesos de remoción en masa, análisis del caso ladera sur de Tuxtla Gutiérrez, Chiapas. Investigaciones Geograficas, 2017(92), 12. https://doi.org/10.14350/rig.52822

Pourghasemi, H. R., Teimoori Y.Z., Panagos, P., y Pradhan, B. (2018). Analysis and evaluation of landslide susceptibility: a review on articles published during 2005-2016 (periods of 20052012 and 2013-2016). Arabian Journal of Geosciences, 11(9), 193. https://doi.org/10.1007/s12517-018-3531-5

Quesada-Román, A., Moncada-López, R., Paz-Tenorio, J. A., Espinoza-Jaime, E., GutiérrezGutiérrez, C., Castellón-Meyrat, A., \& Acosta-Galeano, N. (2018). Las investigaciones sobre movimientos de laderas en Costa Rica, Honduras, México y Nicaragua: enseñanzas desde la academia, las agencias de cooperación y las instituciones públicas. Revista Geográfica de América Central, 1(60), 17. https://doi.org/10.15359/rgac.60-1.1

Quesada-Román, A., \& Zamorano-Orozco, J. J. (2019). Zonificación de procesos de ladera e inundaciones a partir de un análisis morfométrico en la cuenca alta del río General, Costa Rica. Investigaciones Geográficas, 99, 1-19. https://doi.org/10.14350/rig.59843

Ramirez-Cruz, H., Lopez-Velasco, O., e Ibañez-Castillo, L. (2015). Estimation of Monthly 30 Minute RainfallIntensity Based on Pluviometric Data. Terra Latinoamericana , 33(2), 151159. http://www.scielo.org.mx/pdf/tl/v33n2/2395-8030-tl-33-02-00151.pdf 
Reichenbach, P., Rossi, M., Malamud, B., Mihir M., y Guzzetti, F. (2018). A review of statisticallybased landslide susceptibility models. Earth-Science Reviews, 180 (2018), 60-91. https://doi.org/10.1016/j.earscirev.2018.03.001

Riddell, G.A., van Delden, H., Maier, H.R., y Zecchin, A.C. (2019). Exploratory scenario analysis for disaster risk reduction: Considering alternative pathways in disaster risk assessment. International Journal of Disaster Risk Reduction, $39,101230$. https://doi.org/10.1016/j.ijdrr.2019.101230

Saaty, T. L. (1977). A scaling method for priorities in hierarchical structures. Journal of Mathematical Psychology, 15(3), 234-281. https://doi.org/10.1016/0022-2496(77)90033-5

SEDUVI y Delegación Álvaro Obregón. (2011). Programa delegacional de Desarrollo Urbano de Álvaro obregón. In Secretaria de Desarrollo Urbano y Vivienda -SEDUVI. http:// http://www.data.seduvi.cdmx.gob.mx/portal/docs/programas/PDDU_Gacetas/2015/PDDU_ ALVARO-OBREG\%C3\%93N.pdf

Shano, L., Raghuvanshi, T.K., y Meten, M. (2020). Landslide susceptibility evaluation and hazard zonation techniques - a review. Geoenviron Disasters 7, (18). https://doi.org/10.1186/s40677020-00152-0

Servicio Meteorológico Nacional (SMN). (2010). Registros de precipitación diaria CDMX 19982009. [Base de datos no publicada].

Servicio Geológico Mexicano (SGM). (2002). Carta Geológico -Minera; Ciudad de México E142. https://bit.ly/3acLEHz

Universidad Nacional Autónoma de México; Secretaría de Protección Civil de la Ciudad de México (SPC-CDMX). (2014). Atlas de peligros y Riesgos de la Ciudad de México. Actualización de los mapas de riesgo http://atlas.cdmx.gob.mx/mapas/MR_Alvaro_Obregon.pdf

Vojteková J., y Vojtek M. (2020). Assessment of landslide susceptibility at a local spatial scale applying the multi-criteria analysis and GIS: a case study from Slovakia. Geomatics, Natural Hazards and Risk, 11(1), 131-148. https://doi.org/10.1080/19475705.2020.1713233

Xiao, T., Segoni, S., Chen, L., Yin, K., y Casagli, N. (2020). A step beyond landslide susceptibility maps: a simple method to investigate and explain the different outcomes obtained by different approaches. Landslides, 17(3), 627-640. https://doi.org/10.1007/s10346-019-01299-0

\section{Software}

START_LICENSE /SERVER MAFE_IGG ANY 27001

$\begin{array}{lllllll}\text { ARCGIS FEATURE } & \text { Virtual } & \text { EarthEng } & \text { ARCGIS } & 10.3 & \text { 01-jan-2022 }\end{array}$ vendor_info=7KNJDRHFHBK4CFDMJ214 SIGN="052E ABFC 32DD 12473 DEFD E276 4BF3 E0DB 87EB 2203 5A30 C014 19A1 C35E 2154 I08B1 9460 A2B9 6701 DC4D CAF2 E2FE 1347 0E36 90FA 4F3B E864 \BEC8 D3A2 A615" 